\title{
Solvation Influences Flap Collapse in HIV-1 Protease
}

\author{
Kristin L. Meagher and Heather A. Carlson* \\ Department of Medicinal Chemistry, College of Pharmacy, University of Michigan, Ann Arbor, Michigan
}

\begin{abstract}
HIV-1 protease (HIVp) is an important target for the development of therapies to treat AIDS and is one of the classic examples of structurebased drug design. The flap region of HIVp is known to be highly flexible and undergoes a large conformational change upon binding a ligand. Accurately modeling the inherent flexibility of the HIVp system is critical for developing new methods for structurebased drug design. We report several 3-ns molecular dynamics simulations investigating the role of solvation in HIVp flap rearrangement. Using an unliganded crystal structure of HIVp, other groups have observed flap reorganization on the nanosecond timescale. We have also observed rapid, initial flap movement, but we propose that it may be caused by system setup. The initial solvation of the system creates vacuum regions around the protein that may encourage large conformational deformities. By reducing the vacuum space created by the solvation routine, the observed flap collapse is attenuated. Also, a more thorough equilibration procedure preserves a more stable protein conformation over the course of the simulation. Proteins 2005;58:119-125.

๑) 2004 Wiley-Liss, Inc.
\end{abstract}

Key words: molecular dynamics simulation; AMBER; cavity water; system setup; protein flexibility; calculation

\section{INTRODUCTION}

HIV-1 protease (HIVp) is one of the most extensively studied enzymes, both experimentally and computationally. HIVp is an aspartic protease with homology to the mammalian aspartic proteases pepsin and renin. It is active as a homodimer with a $\mathrm{C} 2$ axis of symmetry in the unbound state and contains two Asp-Thr-Gly active-site triads. ${ }^{1}$ HIVp cleaves the viral gag-pol polyprotein into individual proteins and is required for proper assembly of viral progeny. Inhibition of HIVp results in the production of viral particles that are unable to infect other cells. ${ }^{2}$ Due to its critical role in the viral life cycle, HIVp is an attractive target for the development of AIDS therapies, ${ }^{3}$ and 7 drugs are currently marketed as HIVp inhibitors for AIDS treatment. Unfortunately, resistance to these drugs has developed rapidly. ${ }^{4}$ Thus, the design of HIVp inhibitors with novel mechanisms of action and reduced resistance liabilities is still desirable.

The catalytic residues of HIVp are located at the base of a large open cavity, the ceiling of which is composed of 2 flaps- 1 from each monomer (Fig. 1). Upon ligand binding, these flaps move 5-7 $\AA$ toward the base of the cavity, closing in on the ligand, as is observed in all ligand-bound crystal structures. ${ }^{5}$ In contrast to the bound structures, the crystal structure of unbound HIVp has been solved with the flaps in a semiopen conformation. Early molecular dynamics (MD) simulations proposed that the semiopen flap structure found in the unliganded crystal structures was due to crystal packing effects, and that a closed flap structure, like that of the bound structures, was likely the predominant species found in solution. ${ }^{6,7}$ In contrast, researchers who conducted a recent 10-ns MD simulation of the unbound HIVp observed that a novel open-flap conformation is achieved and maintained after approximately 3 ns. $^{8}$ The structures were unique because no open-flap structures have been observed experimentally, although they are necessary to allow substrates to enter the active site. Free energy calculations have been performed on uncomplexed HIVp starting from the semiopen flap conformation, moving to the closed-flap conformation, as seen in ligand-bound structures. ${ }^{9}$ The computed free energy of the flap transition predicts that the semiopen flap conformation is approximately $7 \mathrm{kcal} / \mathrm{mol}$ more stable than the closed-flap conformation without a bound ligand, and that entropic freedom of the semiopen state in solution is responsible for the free energy difference.

Several NMR studies have appeared that detail the dynamics of the flap region. ${ }^{10,11}$ They propose that the flaps are in rapid equilibrium among an ensemble of semiopen conformations, which is in slow equilibrium with open conformations (allowing the ligand access to the active site) and closed-flap conformations. The NMR data suggest that the tips of the flaps (residues 49-53) move among this ensemble of semiopen states on the 1-ns timescale. Both order parameters and chemical shift data indicate that the flap bodies (residues 45-47) maintain a $\beta$-sheet conformation during the NMR experiments, sug-

The Supplementary Materials referred to in this article can be found at http://www.interscience.wiley.com/jpages/0887-3585/suppmat/ index.html

Grant sponsor: National Institutes of Health; Grant number: GM65372. Grant sponsor: Beckman Young Investigator Program. Grant sponsor: Fellowships from Edward S. Blake, Fred W. Lyons, AFPE, and the University of Michigan Regents (to Kristin L. Meagher). Grant sponsor: Pharmacological Sciences Training Program; Grant number: GM07767 NIGMS.

*Correspondence to: Heather A. Carlson, Department of Medicinal Chemistry, College of Pharmacy, University of Michigan, 428 Church St., Ann Arbor, MI 48109-1065. E-mail: carlsonh@umich.edu

Received 28 January 2004; Accepted 25 June 2004

Published online 1 November 2004 in Wiley InterScience (www.interscience.wiley.com). DOI: 10.1002/prot.20274 
$\mathbf{A}$

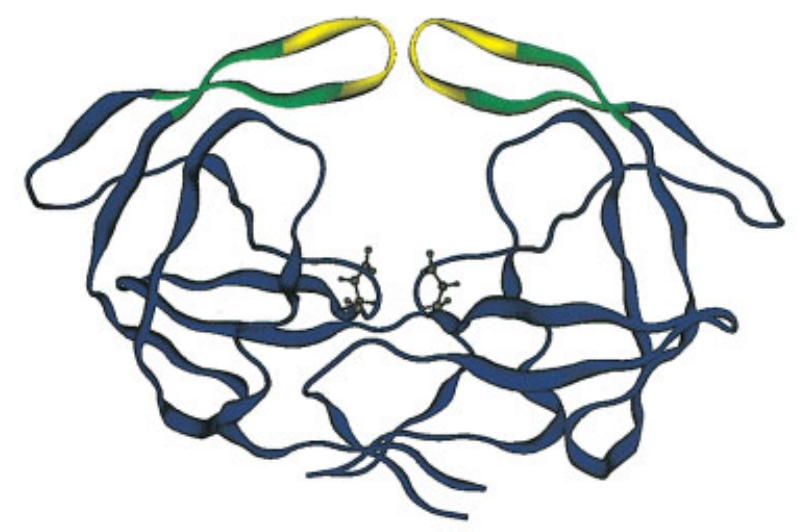

B

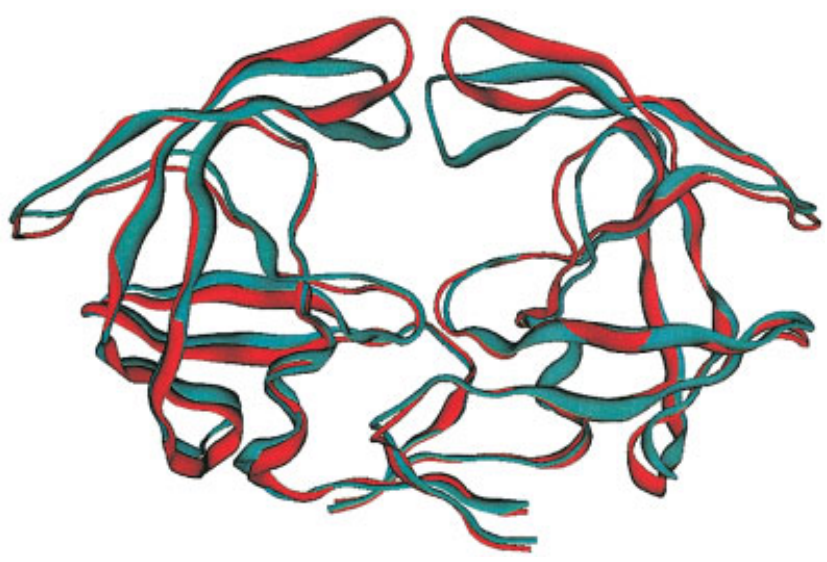

Fig. 1. (A) 1HHP crystal structure of HIVp. The catalytic aspartates from each monomer are shown in ball-and-stick configuration. The flap region (residues $45-56$ from each monomer) is shown in green and the flap tips (residues 49-53) in yellow. Structures are aligned by C $\alpha$. (B) Initial flap collapse after $120-p s$ equilibration. The $1 \mathrm{HHP}$ structure is shown in red, and the collapsed structure in cyan.

gesting less conformational flexibility on the subnanosecond timescale. ${ }^{11}$

Our initial MD simulations of HIVp showed a dramatic conformational change in the flap region after an equilibration time of only 120 ps (Fig. 1). The flaps collapsed approximately $5 \AA$ into the active site and remained collapsed in the subsequent simulation. Such a large-scale motion is not physically realistic on such a short timescale. Since accurate simulations of the flap region of HIVp are critical for structure-based drug design, we sought to understand the observed flap collapse and its implication for the conformational flexibility of the HIVp enzyme.

The solvation algorithm in AMBER6 ${ }^{12}$ superimposes the solute in a pre-equilibrated box of TIP3 $\mathrm{P}^{13}$ water. The distance between the solute and solvent atoms is calculated and compared to the sum of the van der Waals radii. If any solvent atom is closer than this distance, the entire
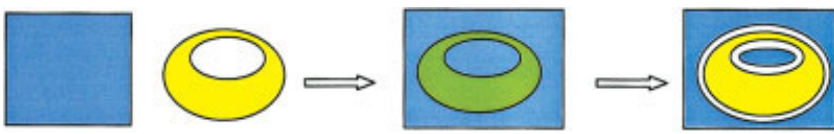

Fig. 2. Cartoon of protein superposition into a solvent box, showing the creation of a small vacuum layer. In the LEAP module of AMBER6, the closeness parameter controls how close the solvent is allowed to the protein.

solvent molecule is removed. This creates a thin vacuum layer around the solute (Fig. 2). For globular proteins, this vacuum layer should pose few problems with the simulation. The equilibration phase will adjust the water packing, and no adverse structural effects should be observed. However, HIVp has a large active-site cavity that is solvent-exposed on 2 sides and covered by 2 thin $\beta$-strand flaps. We propose that in our initial simulations, the flaps of HIVp collapse to alleviate the initial vacuum layer and that the protein collapse occurs before an appropriate amount of water has a chance to enter the active-site cavity.

To understand the importance of initial solvation, a series of simulations were run to probe the role of solvation in flap collapse. By changing the number of water molecules removed during the initial solvation, the flap collapse is attenuated. Similarly, adding an additional preequilibration of the water alone (protein held fixed) also prevents the initial flap collapse and provides a stable protein conformation over the course of the simulation. It should be noted that pre-equilibration of solvent is standard practice in modern MD simulations; however, to our knowledge, there have been no reports of the consequences of inappropriate solvation on large, open active-site cavities.

\section{COMPUTATIONAL METHODS}

The coordinates for the unliganded monomer of HIVp were obtained from the Protein Data Bank ${ }^{14}$ (PDB accession code: $1 \mathrm{HHP}^{1}$ ) and the homodimer was generated via symmetry operations. Hydrogens were added with the program MOE. ${ }^{15}$ The hydrogens were minimized using the AMBER94 force field ${ }^{16}$ as implemented in MOE. The side chains were allowed to relax by minimizing iteratively in stages out from the active site. The minimization protocol consisted of steepest descent steps to a root-mean-square (RMS) gradient of $10 \mathrm{kcal} / \mathrm{mol} \cdot \AA$, conjugate gradient steps to a gradient of $1 \mathrm{kcal} / \mathrm{mol} \cdot \AA$, and 200 truncated-Newton steps or a convergence of $0.001 \mathrm{kcal} / \mathrm{mol} \cdot \AA$. The $+4 e$ charge of HIVp was neutralized by the inclusion of 4 chloride counterions. The electrostatics module in $\mathrm{MOE}$ was used to calculate the potential and place the counter ions iteratively $10 \AA$ from the protein surface in the most electropositive regions.

Several different methods of solvation were used in setup. The initial studies used the default solvation algorithm implemented in the LEAP module of the AMBER6 ${ }^{12}$ suite of programs to generate a $62 \times 62 \times 82 \AA^{3}$ box of TIP3P ${ }^{13}$ water. In the solvation algorithm, the closeness parameter controls the minimum distance between the 
solvent molecules and the protein. To determine close contacts, the sum of the van der Waals radii for 2 atoms is multiplied by the closeness parameter $(\mathrm{C})$ and compared to the distance between the atoms. Thus, by decreasing the magnitude of the closeness parameter, more solvent molecules are retained closer to the protein surface. Changing this parameter does not affect the density of the bulk of the water box, only the number of solvent molecules that are removed at the protein surface. Three additional systems were created using different values of C: 1.0, 0.5, and 0.25. To vary the initial number of waters placed in the activesite cavity, a cap of solvent with the desired closeness was centered on the flap region. We performed 10,000 steps of minimization of the water and protein hydrogens using sander_classic. The resulting structure was placed in a standard TIP3P water box of $62 \times 62 \times 82 \AA^{3}$, and counterions were reintroduced to ensure a net-neutral simulation. This system was minimized an additional 10,000 steps with the protein heavy atoms fixed (first 10 steps of steepest descent for coarse corrections and then 9990 steps of conjugate gradient minimization).

A fourth simulation of $1 \mathrm{HHP}$ used the standard water setup $(C=1)$; however, the protein was held fixed during the initial warming to $298 \mathrm{~K}(50 \mathrm{ps})$ and equilibration (200 ps) to allow the water to optimally complement the surfaces and cavities of the frozen protein structure. A second warming and equilibration (as described below) was then performed with no restraints.

MD simulations were performed with the AMBER94 force field ${ }^{16}$ and the sander_classic module in the AMBER6 ${ }^{12}$ suite of programs. For all simulations, the temperature was raised to $298 \mathrm{~K}$ over $50 \mathrm{ps}$ in 10-ps intervals, followed by an equilibration phase of $200 \mathrm{ps}$. The simulation was run in the NPT ensemble, and $\mathrm{SHAKE}^{17}$ was used to restrain hydrogen bonds lengths. A 2-fs timestep was used, along with a $10-\AA$ cutoff for nonbonded interactions. The nonbonded pair list was updated every 15 steps and particle mesh Ewald ${ }^{18}$ (PME) was used for long-range electrostatics. The production phase of the MD simulations was $3 \mathrm{~ns}$ for the 4 different solvation routines.

Root-mean-square deviation (RMSD) traces were calculated using the carnal module of the AMBER6 suite of programs based on the $\mathrm{C} \alpha$ positions of the $1 \mathrm{HHP}$ crystal structure. Order parameters were calculated from the MD trajectory using the ptraj module of the AMBER suite to calculate the autocorrelation function for each N-H internuclear vector. The resulting correlation curves were fit to the Lipari-Szabo "model-free" equation. ${ }^{19} \mathrm{C} \alpha$ RMS fluctuations were also calculated for the 3 -ns trajectory using ptraj.

Cavity waters were quantized by defining a sphere to represent the active-site volume for each model. A reasonable and consistent means of evaluating cavity size was needed that was independent of the large conformational changes observed. The center of the cavity was determined by averaging the $\mathrm{C} \alpha$ positions of residues that lined the active site $\left(23-32,47-54,84,23^{\prime}-32^{\prime}, 47^{\prime}-54^{\prime}, 84^{\prime}\right)$. The radius of the spherical cavity was the average of the distances from the center to the $\mathrm{C} \alpha$ positions of the
$\mathbf{A}$

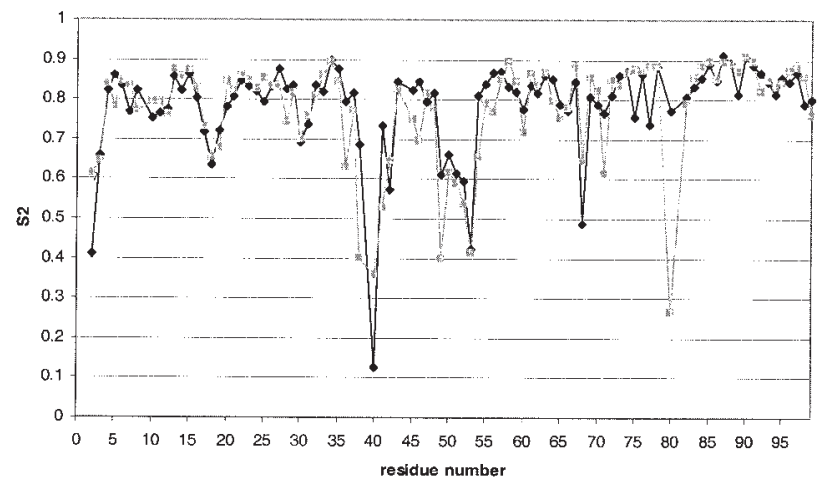

B

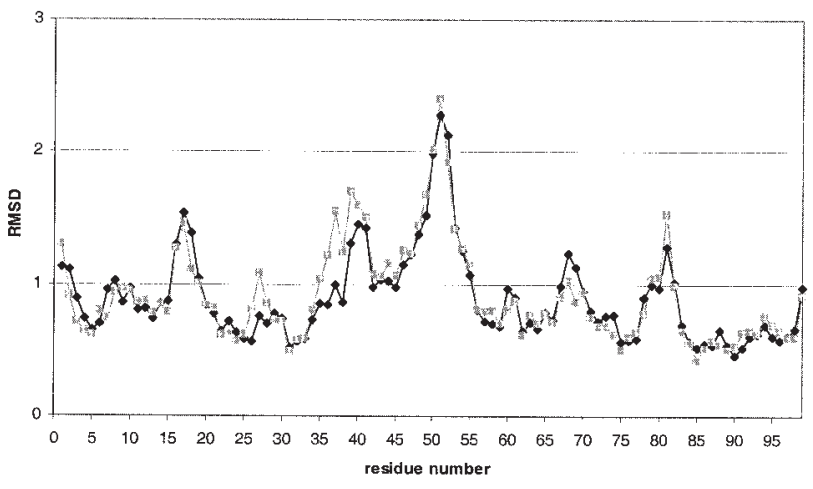

Fig. 3. (A) Calculated order parameters $\left(\mathrm{S}^{2}\right)$ for the $\mathrm{N}-\mathrm{H}$ internuclear vector over $3 \mathrm{~ns}$ of the pre-equilibrated trajectory. (B) C $\alpha$ RMSD fluctuations in angstrom units. The two monomers are shown together: residues $2-99$ in black and $2^{\prime}-99^{\prime}$ in gray.

selected residues. As the protein structure relaxed during the simulations, the center and radii changed according to the amount of conformational change. Waters inside this sphere were considered cavity water.

\section{RESULTS AND DISCUSSION Agreement With NMR Data}

Using a properly equilibrated system, we see good agreement with recent experimental data. The simulation with the pre-equilibrated water shows motion that is consistent with the NMR data, ${ }^{10,11}$ indicating that the HIVp flaps are sampling an ensemble of states around the semiopen crystal conformation. To compare our simulation with existing NMR data, we calculated both order parameters for the internuclear N-H vector and $\mathrm{C} \alpha$ atomic fluctuations over $3 \mathrm{~ns}$ of our trajectory. Comparing calculated and experimental order parameters can be problematic; however, qualitative trends are generally consistent. ${ }^{20}$ The calculated order parameters and RMSD fluctuations for each monomer are summarized in Figure 3. Freedberg et al. ${ }^{11}$ found that only residues $37-41$ (flap elbows), 49-53 (flap tips), and 80 of HIVp have order parameters $<0.75$. Our calculated values follow this same trend of low-order parameters only in these same regions. The residues 
43-48 (flap bodies) have high order parameters and low RMSD fluctuations, which again agree with the NMR results that these residues maintain a $\beta$-sheet conformation on the timescale investigated. There are small differences between the 2 monomers, illustrating the limitations of a comparing a single MD trajectory to the averaging obtained in an NMR experiment. The agreement between the calculated and experimental order parameters indicates that our pre-equilibrated simulation is sampling appropriate conformations on the nanosecond timescale.

For the simulation with pre-equilibrated water, we do not see the dramatic flap reorganization reported by Scott and Schiffer. ${ }^{8}$ They point out that there are important crystal contacts in the 1HHP structure, and it is very possible that these interactions stabilize the semiopen form of HIVp. They make the intriguing argument that without these contacts in the MD simulation, the semiopen form is no longer stable. An unstable protein conformation should change over the course of an MD simulation, and they hypothesize that this is the cause of the large flap rearrangement seen within $3 \mathrm{~ns}$ of their simulation.

However, our simulations also lack those crystal contacts, yet the protein conformation remains stable. The exact cause for the differences in our simulation results is unclear. First and foremost, their simulations were done with another excellent MD software package, GROMOS. ${ }^{21}$ Differences are inherent when using another code and force field. The AMBER94 force field has a very slight tendency to favor $\alpha$-helices over extended sheets, ${ }^{22-24}$ so one might expect that our simulations would be biased toward a folding of the $\beta$-sheet flaps. Since we did not see this with pre-equilibrated water, we feel this is strong support for the stability of the semiopen form seen in the crystal structure. New work by McCammon and coworkers has recently appeared, examining the conformational behavior of wild-type and mutant forms of HIVp. ${ }^{25}$ They started with closed forms of the protein, removed the ligands, and ran 22-ns MD simulations using AMBER. In that length of time, both the wild-type and mutant HIVp sampled closed and semiopen forms with large degrees of flexibility seen in the flap tips, but opening of the flaps was not observed.

It is possible that the flap rearrangement seen by Scott and Schiffer ${ }^{8}$ may be due to the issue of solvating the cavity. We observe large conformational changes in the flaps with under- or oversolvation of the binding-site cavity (see below). Their paper states that they initiated their MD simulations at $300 \mathrm{~K}$, with harmonic constraints on the protein atoms, and removed those constraints after only $10 \mathrm{ps}$. This short time would not have allowed much water to enter or escape the binding site if needed, if pre-equilibration was not performed. We are not implying that Scott and Schiffer's simulation was flawed. It is very common for setup to include pre-equilibration of the water but not be explicitly reported in the paper. Furthermore, we show below that a tighter packing of initial waters provides a stable simulation $(\mathrm{C}=0.5)$. If the initial solvation protocol in GROMOS places more waters in the cavity, the influence of vacuum around the protein would obviously be reduced.

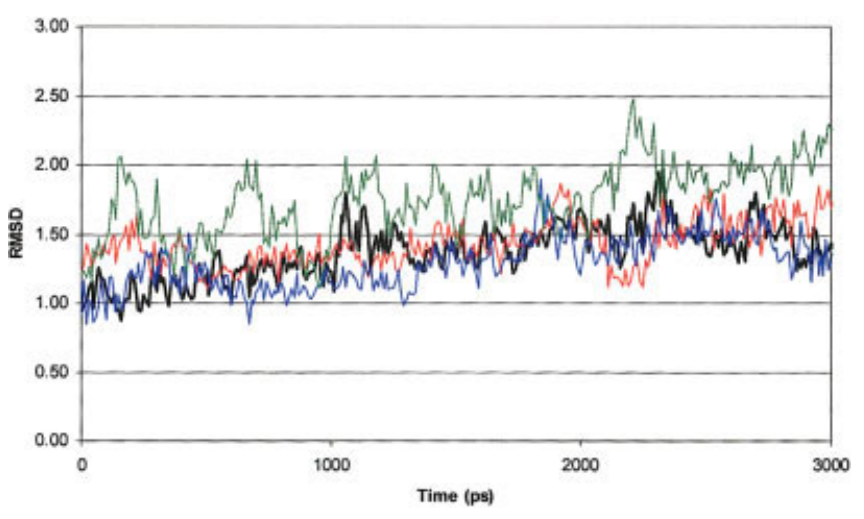

Fig. 4. RMSD traces for the 4 simulations: black is pre-equilibrated water, green is $C=0.25$, blue is $C=0.5$, and red is $C=1.0$.

TABLE I. Number of Water Molecules in the HIVp Active-Site Cavity After the Initial Solvation and Following 200-ps Equilibration

\begin{tabular}{lcc}
\hline System & $\begin{array}{c}\text { Number of Initial } \\
\text { Cavity Waters }\end{array}$ & $\begin{array}{c}\text { Number of Cavity Waters } \\
\text { After 200-ps Equilibration }\end{array}$ \\
\hline $\mathrm{C}=1.0$ & 33 & 49 \\
$\mathrm{C}=0.50$ & 79 & 80 \\
$\mathrm{C}=0.25$ & 137 & 130 \\
\hline
\end{tabular}

\section{Comparing Initial Solvation Conditions}

Simulations using 4 different solvation setups were examined over the course of $3 \mathrm{~ns}$ of simulation. Three simulations were run varying the "closeness" parameter (C) in the solvation routine in AMBER6 (see Methods section) and a fourth simulation used an additional, initial pre-equilibration phase, where the protein was held fixed and the solvent was allowed to relax around it (not performed in the $\mathrm{C}=1.0, \mathrm{C}=0.5$, and $\mathrm{C}=0.25$ simulations). The RMSD traces for the simulations are shown in Figure 4, and the potential energy traces are provided as Supplementary Information. All 4 simulations exhibit proper equilibration and stable potential energy traces over the course of the 3 -ns simulation.

The RMSD from the crystal structure $\mathrm{C} \alpha$ positions stabilizes at $\sim 1.5 \AA$ A for the pre-equilibrated water simulation. The RMSD traces for the simulations with different closeness parameters show more variation. The RMSD trace for the $\mathrm{C}=0.5$ simulation most closely resembles the pre-equilibrated water trace. In contrast, the $\mathrm{C}=0.25$ simulation shows a constant trend away from the crystal position. It is interesting to observe that RMSD trace for the $\mathrm{C}=1$ simulation (default solvation) initially diverges but at approximately $2 \mathrm{~ns}$ returns to a structure similar to the starting conformation.

The number of water molecules present in the active-site cavity can be quantified and compared among the 4 simulations (Tables I and II). As expected, the initial amount of water in the cavity varies with $\mathrm{C}$ and directly reflects the amount of vacuum created around the binding site. There is a significant difference between the $\mathrm{C}=1.0$ and the $\mathrm{C}=0.25$ simulations, with 33 and 137 water 
TABLE II. Number of Water Molecules in the HIVp ActiveSite Cavity After the Initial Solvation, Pre-Equilibration of Water, and Following an Additional 200-ps Equilibration

\begin{tabular}{|c|c|c|c|}
\hline System & $\begin{array}{c}\text { Number of } \\
\text { Initial } \\
\text { Cavity } \\
\text { Waters } \\
\end{array}$ & $\begin{array}{c}\text { Number of } \\
\text { Cavity } \\
\text { Waters } \\
\text { After Pre- } \\
\text { Equilibration }\end{array}$ & $\begin{array}{c}\text { Number of } \\
\text { Cavity } \\
\text { Waters } \\
\text { After 200- } \\
\text { ps Full } \\
\text { Equilibration }\end{array}$ \\
\hline Pre-equilibrated & 27 & 85 & 89 \\
\hline
\end{tabular}

molecules in the respective cavities. After 200 ps of allatom equilibration, the amount of water in the active-site cavity is approximately the same as the initial solvation (Table I); however, in the $\mathrm{C}=1.0$ and $\mathrm{C}=0.25$ simulations, the protein has deformed significantly (Fig. 5). For $\mathrm{C}=1.0$, slightly more water is seen entering the cavity. The large flap motion seen in the $C=0.25$ and $C=1.0$ simulations indicates that protein deformation occurs before bulk water has a chance to enter or leave the cavity.

Table II demonstrates the importance of pre-equilibrating the water while holding the protein fixed. The initial solvation places 27 waters in the active site. After preequilibration in which the protein is held frozen, there are 85 waters in the cavity! A difference of 58 TIP3P water molecules is a little more than $1700 \AA^{3}{ }^{13}$ It should be noted that the protein was held fixed, so identical spheres were used to determine the number of waters. The increase observed is not from any deformation of the protein, but comes from diffusion of water molecules into an undersolvated cavity. After an additional 200 ps of equilibration, where the protein has also been allowed to relax, 89 waters are observed in the cavity.

Since the number of cavity waters changed only slightly after the second equilibration, pre-equilibrating the water appears to create stable and appropriate solvation of the active-site cavity. Comparing the three C-dependent simulations with the pre-equilibrated simulation shows that the $\mathrm{C}=0.5$ case provides the most similar initial solvation, placing 79 waters in the active-site cavity, and, after all-atom equilibration, 80 waters remain in the cavity. It is interesting that similar numbers of water molecules in the $\mathrm{C}=0.5$ case also show a stable simulation similar to the pre-equilibrated case.

Examining snapshots taken along the MD trajectory for all 4 systems illustrates the conformational consequences of the different solvation models (Fig. 5). The $\mathrm{C}=1.0$ system shows significant collapse of the flap region after $200 \mathrm{ps}$ of equilibration. However, the pre-equilibrated water system and the $\mathrm{C}=0.5$ system do not exhibit this collapse and instead maintain the crystal structure conformation (Fig. 5). It is interesting that the flaps appear to be opening in the $\mathrm{C}=0.25$ simulation, perhaps due to too many water molecules in the active-site cavity. Over the course of the 3 -ns simulation, the pre-equilibrated and $\mathrm{C}=$ 0.5 systems retain their crystal structure conformation, as can be seen in the stable RMSD traces of Figure 4 and the snapshots from the simulations shown in Figure 5.
These observations are consistent with our hypothesis regarding the influence of solvation on conformation. Allowing the water to pack extremely close to the protein $(\mathrm{C}=0.25)$ results in a diverging RMSD trace due to large motions as the flaps move to accommodate the extra water. The protein structure has changed rapidly and dramatically, indicating that the $\mathrm{C}=0.25$ simulation does not represent realistic solvation of the HIVp active site. Conversely, using the default parameter $(\mathrm{C}=1.0)$ initially results in inadequate solvation of the active-site cavity and flap collapse. However, extending the simulation to $2 \mathrm{~ns}$, the $\mathrm{C}=1.0$ simulation samples conformations more closely related to the semiopen flap structure seen in the crystal structure. This is consistent with the previous free energy calculation, ${ }^{9}$ indicating that the semiopen flap structures are more favorable than closed flaps for unliganded HIVp. The most appropriate solvation is observed in the $\mathrm{C}=0.5$ and pre-equilibrated systems. These systems show appropriate, stable dynamics-sampling an ensemble of semiopen conformations. Certainly, pre-equilibration of the water is most appropriate and leads to the most realistic simulations. This is why it is the current standard in MD simulations; however, we have demonstrated the potential conformational consequences of inappropriate solvation - both over- and undersolvation - of a large cavity.

By investigating the effect of the closeness parameter, we have found that flap motion is dependent on the solvation of the active-site cavity, particularly in the early phases of the simulation. By manipulating the number of waters retained in the active site, we observe a range of flap conformations.

\section{CONCLUSIONS}

Using a properly equilibrated system, we see good agreement with recent NMR experiments. This indicates that our system is sampling appropriate conformations on the nanosecond timescale. We are not able to reproduce the large rearrangements seen by Scott and Schiffer. ${ }^{8}$ It is possible that this disagreement is the result of our chosen software package and force field, but it may be related to the solvation of the binding site.

Appropriate solvation of proteins with large cavities is critical for reasonable conformational behavior in MD simulations. For HIVp, the layer of vacuum created with the standard solvation routine can cause the flaps to collapse into the active-site cavity, leading to a less natural trajectory. By decreasing the cutoff distance between solvent molecules and the solute, solvent is allowed to pack closer to the protein surface, lessening the effects of the vacuum layer. Using a series of simulations, we have shown that including either too much $(C=0.25)$ or not enough $(\mathrm{C}=1.0)$ water in the HIVp active-site cavity leads to unrealistic protein conformations early in the simulation. Using an intermediate value $(\mathrm{C}=0.5)$, or holding the protein fixed and equilibrating the water around the protein structure, provides appropriate solvation and produces a stable trajectory. [Supporting material including the potential energy traces for the four simulations is available.] 


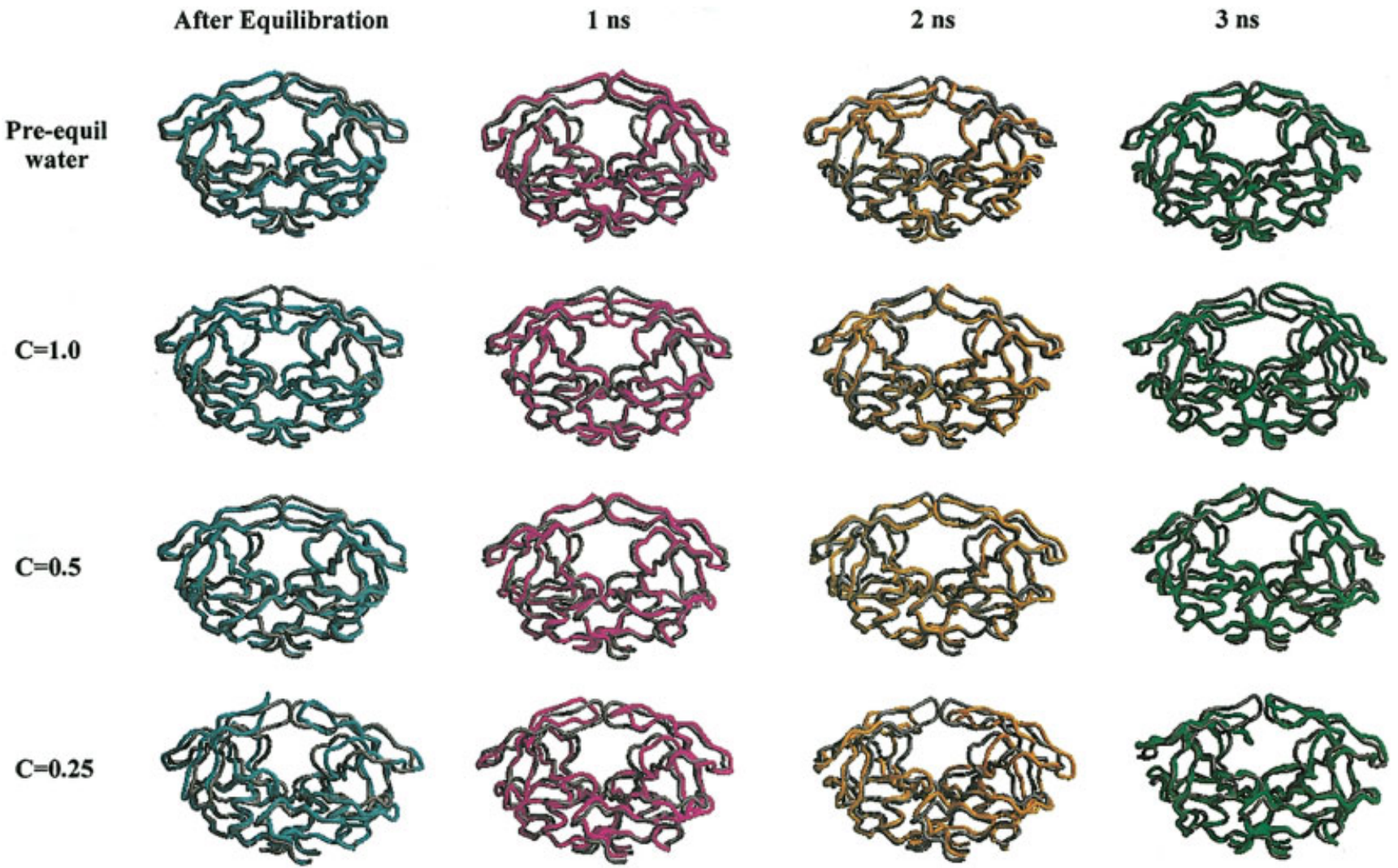

Fig. 5. Protein conformations taken from the $4 \mathrm{MD}$ simulations. All structures are aligned to the $1 \mathrm{HHP} \mathrm{C} \alpha$ coordinates from the crystal structure (shown in gray). The equilibrated structures are shown in cyan; conformation after $1 \mathrm{~ns}$, in magenta; after $2 \mathrm{~ns}$, in orange; and after $3 \mathrm{~ns}$, in green.

\section{ACKNOWLEDGMENTS}

Our thanks to Michael Lerner for the Python scripts used to calculate the NMR order parameters.

\section{REFERENCES}

1. Spinelli S, Liu QZ, Alzari PM, Hirel PH, Poljak RJ. The threedimensional structure of the aspartyl protease from the HIV-1 isolate BRU. Biochimie 1991;73:1391-1396.

2. Kohl NE, Emini EA, Schleif WA, Davis LJ, Heimbach JC, Dixon RAF, Scolnick EM, Sigal IS. Active human immunodeficiency virus protease is required for viral infectivity. Proc Natl Acad Sci USA 1988;85:4686-4690

3. Wlodawer A, Vonderasek J. Inhibitors of HIV-1 protease: a major success of structure-assisted drug design. Annu Rev Biophys Biomol Struct 1998;27:249-284.

4. Boden D, Markowitz M. Resistance to human immunodeficiency virus type 1 protease inhibitors. Antimicrob Agents Chemother 1998;42:2775-2783.

5. Wlodawer A, Gustchina A. Structural and biochemical studies of retroviral proteases. Biochim Biophys Acta 2000;1477:16-34

6. Harte WE, Swaminathan S, Mansuri MM, Martin JC, Rosenberg IE, Beveridge DL. Domain communication in the dynamical structure of human immunodeficiency virus 1 protease. Proc Natl Acad Sci USA 1990;87:8864-8868.

7. York DM, Darden TA, Pedersen LG, Anderson MW. Molecular dynamics simulation of HIV-1 protease in a crystalline environment and in solution. Biochemistry 1993;32:1443-1453.

8. Scott WRP, Schiffer CA. Curling of flap tips in HIV-1 protease as a mechanism for substrate entry and tolerance of drug resistance. Structure 2000;8:1259-1265.

9. Rick SW, Erickson JW, Burt SK. Reaction path and free energy calculations of the transition between alternate conformations of HIV-1 protease. Proteins 1998;32:7-16.
10. Ishima R, Freedberg DI, Wang Y-X, Louis JM, Torchia DA. Flap opening and dimer-interface flexibility in the free and inhibitorbound HIV protease, and their implications for function. Structure 1999;7:1047-1055.

11. Freedberg DI, Ishima R, Jacob J, Wang Y-X, Kustanovich I, Louis JM, Torchia DA. Rapid structural fluctuations of the free HIV protease flaps insolution: relationship to crystal structures and comparison with predictions of dynamics calculations. Prot Sci 2002;11:221-232.

12. Case DA, Pearlman DA, Caldwell JW, Cheatham III TE, Ross WS, Simmerling CL, Darden TA, Merz KM, Stanton RV, Cheng AL, Vincent JJ, Crowley M, Tsui V, Radmer RJ, Duan Y, Pitera J, Massova I, Seibel GL, Singh UC, Weiner PK, Kollman PA. AMBER 6. San Francisco: University of California at San Francisco; 1996.

13. Jorgensen WL, Chandrasekhar JD, Madura RW, Impey RW, Klein ML. Comparison of simple potential functions for simulating liquid water. J Chem Phys 1983;79:926-935.

14. Berman HM, Westbrook J, Feng Z, Gilliland G, Bhat TN, Weissig N, Shindyalov IN, Bourne PE. The Protein Data Bank. Nucleic Acids Res 2000;28:235-242.

15. Molecular Operating Environment. Montreal, Canada: Chemical Computing Group, Inc.; 2001.

16. Cornell WD, Cieplak P, Bayly CI, Gould IR, Merz KM, Ferguson DM, Spellmeyer DC, Fox T, Caldwell JW, Kollman PA. A second generation force field for the simulation of proteins, nucleic acids, and organic molecules. J Am Chem Soc 1995;117:5179-5197.

17. Ryckaert JP, Ciccitti G, Berendsen HJC. Numerical-integration of Cartesian equations of motion of a system with constraintsmolecular dynamics of $N$-alkanes. J Comp Phys 1977;23:327-341.

18. Darden TA, York D, Pedersen L. Particle mesh Ewald: an $N$ - $\log (N)$ method for Ewald sums in large systems. J Chem Phys 1993;98: 10089-10092.

19. Lipari G, Szabo A. Model-free approach to the interpretation of 
nuclear magnetic resonance relaxation in macromodels: 1 . Theory and range of validity. J Am Chem Soc 1982;104:4546-4559.

20. Case DA. Molecular dynamics and NMR spin relaxation in proteins. Acc Chem Res 2002;35:325-331.

21. Scott WRP. Hünenberger PH, Tironi IG, Mark AE, Billeter SR, Fennen J, Torda AE, Huber T, Krüger P, van Gunsteren WF. The GROMOS biomolecular simulation program package. J Phys Chem A 1999;103:3596-3607.

22. Ponder JW, Case DA. Force fields for protein simulations. Adv Protein Chem 2003;66:27-85.
23. García AE, Sanbonmatsu KY. $\alpha$-Helical stabilization by side chain shielding of backbone hydrogen bonds. Proc Natl Acad Sci USA 2002;99:2782-2787.

24. Simmerling C, Strockbine B, Roitberg AE. All-atom structure prediction and folding simulations of a stable protein. J Am Chem Soc 2002;124:11258-11259.

25. Perryman AL, Lin J-H, McCammon JA. HIV-1 protease molecular dynamics of a wild-type and of the V82F/I84V mutant: possible contributions to drug resistance and a potential new target site for drugs. Protein Sci 2004; 13:1108-1123. 